\title{
Segregation of Amphetamine Reward and Locomotor Stimulation between Nucleus Accumbens Medial Shell and Core
}

\author{
Laurie H. L. Sellings and Paul B. S. Clarke \\ Department of Pharmacology and Therapeutics, McGill University, Montreal, Quebec, Canada H3G 1Y6
}

\begin{abstract}
Convergent evidence suggests that amphetamine (AMPH) exerts its rewarding and locomotor stimulating effects via release of dopamine in the nucleus accumbens. However, there is no consensus as to the relative contributions of core and medial shell subregions to these effects. Moreover, the literature is based primarily on intracranial administration, which cannot fully mimic the drug distribution achieved by systemic administration. In the present study, the effects of bilateral 6-hydroxydopamine lesions of the accumbens core or medial shell on rewarding and locomotor stimulating effects of systemically administered amphetamine $(0.75 \mathrm{mg} / \mathrm{kg}$, i.p. $)$ were examined in a conditioned place preference (CPP) procedure relying solely on tactile cues (floor texture). Residual dopamine innervation was quantified by $\left[{ }^{125} \mathrm{I}\right]-\mathrm{RTI}-55$ binding to the dopamine transporter. When lesions were performed before the conditioning phase, AMPHinduced locomotor stimulation and CPP magnitude were positively correlated with residual dopamine transporter binding in core and medial shell, respectively. Medial shell lesions did not affect morphine CPP, arguing that a sensory or mnemonic deficit was not responsible for the lesion-induced reduction in AMPH CPP. Medial shell lesions performed between the conditioning phase and the test day reduced the expression of amphetamine CPP. These results suggest that after systemic amphetamine administration, rewarding and locomotor stimulating effects of the drug are anatomically dissociated within the nucleus accumbens: the medial shell contributes to rewarding effects, whereas the core contributes to behavioral activation.
\end{abstract}

Key words: nucleus accumbens core; nucleus accumbens medial shell; amphetamine; 6-hydroxydopamine; locomotion; reward; conditioned place preference; morphine

\section{Introduction}

Convergent evidence suggests that the rewarding and behavioral activating effects of psychomotor stimulant drugs are initiated by increased dopaminergic transmission in the nucleus accumbens (NAcc). Evidence is perhaps strongest for the prototypic psychomotor stimulant, amphetamine (AMPH). For example, the locomotor stimulant effect of systemic AMPH is mimicked by intra-accumbens infusion of AMPH or dopamine (DA) (Pijnenburg et al., 1976; Campbell et al., 1997) and is inhibited by intraaccumbens administration of DA antagonists (Pijnenburg et al., 1975; Roberts et al., 1975; Phillips et al., 1994) or 6-hydroxydopamine (6-OHDA) (Kelly et al., 1975; Joyce et al., 1983; Clarke et al., 1988). Similarly, the rewarding effects of AMPH are either mimicked or inhibited by the same types of manipulations (Yokel and Wise, 1976; Lyness et al., 1979; Spyraki

\section{Received March 19, 2003; revised April 23, 2003; accepted May 15, 2003.}

This work was supported by the Natural Sciences and Engineering Research Council of Canada (NSERC) and the Canadian Institutes of Health Research. L.H.L.S holds an NSERC Postgraduate Award, and P.B.S.C. is a Chercheur National of the Fonds de la Recherche en Santé du Québec. We thank Dr. Paul Vezina for advice on conditioned tactile preference, Lindsey McQuade and Robert Biskin for help assembling the test apparatus, Rebecca J. Grant for discussions regarding lesion parameters, and Dr. Sandra M. Boye for comments on this manuscript and help producing figures.

Correspondence should be addressed to Paul B. S. Clarke, Department of Pharmacology and Therapeutics, McGill University, 3655 Promenade Sir-William-0sler Room 1312, Montreal, Quebec, Canada, H3G 1Y6. E-mail: pclarke@pharma.mcgill.ca.

Copyright $\odot 2003$ Society for Neuroscience $\quad 0270-6474 / 03 / 236295-09 \$ 15.00 / 0$ et al., 1982; Carr and White, 1991; Phillips et al., 1994; Izzo et al., 2001).

The NAcc is a heterogeneous structure, as evinced by immunohistochemical staining and neuronal projection patterns (Zahm and Brog, 1992). The major subdivisions are a medioventral shell and a dorsolateral core. These subregions are functionally distinct (Maldonado-Irizarry and Kelley, 1995; Weiner et al., 1996; Kelley et al., 1997; Parkinson et al., 1999; Boye et al., 2001; Ikemoto, 2002), but their precise roles in reward and locomotor activation are uncertain. For example, the locomotor stimulant effect of AMPH has been attributed to an action in the core (Weiner et al., 1996; West et al., 1999; Boye et al., 2001) or in medial shell (Heidbreder and Feldon, 1998; Parkinson et al., 1999 ) or in both structures (Pierce and Kalivas, 1995; Ikemoto, 2002). In contrast, certain dopaminergic drugs have been shown to maintain responding when infused into (medial) shell but not core (Carlezon and Wise, 1996; Ikemoto et al., 1997). Intra-shell self-administration of AMPH has also been reported (Hoebel et al., 1983; Chevrette et al., 2002), but in these studies intra-core infusions were not examined.

A feature of almost all the behavioral studies using AMPH was that the drug was given directly into the NAcc; after intracranial administration, drug distribution and local concentration differ markedly from that achieved after systemic administration. Recently, we combined systemic AMPH administration with 
6-OHDA lesions and found that locomotor stimulation was blunted by dopaminergic denervation of core and not medial shell (Boye et al., 2001).

The present study aimed to establish the relative involvement of NAcc core and medial shell subregions in systemic AMPHinduced behavioral activation and reward. Rats that had sustained 6-OHDA lesions of NAcc core or medial shell were assessed for AMPH-induced locomotor activation and conditioned place preference (CPP). To assess the possibility that decreased $\mathrm{CPP}$ indicated a deficit not in reward but in learning, memory, or sensory function, morphine CPP was also tested.

\section{Materials and Methods}

\section{Subjects}

Subjects were 142 male Long-Evans rats (Charles River, St. Constant, Quebec) weighing 270-310 gm at time of surgery. Rats were housed in groups of three in clear Plexiglas cages in a temperature- and humiditycontrolled animal colony that was lit from 7 A.M. to 7 P.M. Food and water were available ad libitum except during behavioral testing. All experiments were approved by the McGill Faculty of Medicine Animal Care Committee in accordance with Canadian Council on Animal Care guidelines.

\section{Stereotaxic infusion of 6-OHDA}

Rats were anesthetized with ketamine $\mathrm{HCl}(90 \mathrm{mg} / \mathrm{kg}$, i.p.) and xylazine $\mathrm{HCl}(16 \mathrm{mg} / \mathrm{kg}$, i.p.) $15 \mathrm{~min}$ after pretreatment with atropine methyl nitrate $(0.05 \mathrm{mg} / \mathrm{kg}$, s.c.). The rat was placed in a stereotaxic apparatus (Kopf, Tujunga, CA) with the incisor bar set at $-3.9 \mathrm{~mm}$. Rats received bilateral infusions of either 6-OHDA or vehicle into either NAcc core or medial shell. Infusions were made via a 30 gauge stainless steel cannula attached by polyethylene tubing to a $10 \mu \mathrm{l}$ Hamilton syringe driven by a model 5000 Micro Injection Unit (Kopf). For greater accuracy, coordinates for both the core and the medial shell were derived from the mean of two coordinate systems. Thus, anterior-posterior coordinates were $+10.3 \mathrm{~mm}$ from interaural zero and $+1.3 \mathrm{~mm}$ from bregma for both core and shell. Lateral coordinates were $\pm 0.6 \mathrm{~mm}$ (shell) and $\pm 2.4 \mathrm{~mm}$ (core). Ventral coordinates for shell (three injections) were $+2.0,+2.4$, and $+2.8 \mathrm{~mm}$ from interaural zero and $-8.0,-7.6$, and $-7.2 \mathrm{~mm}$ from bregma. Ventral coordinates for core were $+2.9 \mathrm{~mm}$ from interaural zero and $-7.1 \mathrm{~mm}$ from bregma. All coordinates are based on the atlas of Paxinos and Watson (1997). 6-OHDA or vehicle was infused on each side in a volume of $0.1 \mu \mathrm{l}$ (core) or as three infusions of $0.06 \mu \mathrm{l}$ each (shell) at a rate of $0.1 \mu \mathrm{l} / \mathrm{min}$. The concentration of $6-\mathrm{OHDA}$ used was $80 \mu \mathrm{g} / \mu \mathrm{l}$ (core) or $48 \mu \mathrm{g} / \mu \mathrm{l}$ (shell). The cannula remained at the final infusion site for $5 \mathrm{~min}$. Dipyrone (100 mg/kg, s.c.) provided analgesia after surgery. Animals were allowed 7-11 d recovery before conditioning (experiments 1 and 3) or testing (experiment 2). Four animals died after surgery in experiment 3.

\section{Conditioned place preference testing}

General procedure. The method was modified from that of Vezina and Stewart (1987). Eight CPP cages [58.1 cm (length) $\times 28.8 \mathrm{~cm}$ (width) $\times$ $53.0 \mathrm{~cm}$ (height)] were used, each comprising four outer walls made of white plastic-coated particle board (Melamine) and an open top. Cages sat on linoleum flooring covered with a thin layer of Beta Chip bedding. There was no wall dividing the cage into two compartments. Two removable square floor tiles [28.5 cm (length) $\times 28.5 \mathrm{~cm}$ (width) $\times 5.5 \mathrm{~cm}$ (height)] were inserted into each cage; these served as tactile cues. Floor tiles were of two types: mesh and bar. These two textures were provided, respectively, by a stainless steel grid with squares of $1 \times 1 \mathrm{~cm}$ and by 12 stainless steel bars of $1.2 \mathrm{~cm}$ diameter separated by $1.5 \mathrm{~cm}$ edge to edge. Both floor types were mounted on square Melamine frames. All behavioral testing was performed in a room lit with a Kodak GBX-2 safelight filter (Vistek, Toronto, Ontario, Canada) providing far-red illumination (wavelength $>650 \mathrm{~nm}$ ) to minimize visual cues. The location and movements of rats during behavioral testing were monitored by a closed circuit television video camera (Panasonic) linked to a commercial tracking system (EthoVision v3.0, Noldus Information Technology, Leesburg, VA).

Behavioral testing took place over 8 consecutive days and consisted of three phases: preexposure, conditioning, and testing. During all three phases, animals were habituated to the test room in home cages for 15 min before placement into test CPP cages. The preexposure phase served to habituate each animal to the CPP cage itself. This phase comprised a single 20 min session performed in the absence of floor tiles. The conditioning phase took place on days $2-7$. It comprised six daily sessions of 45 min each: three sessions with drug and three sessions with saline administration. Drug and saline were administered on alternating days. After injection, each rat was immediately placed in the middle of a CPP cage. During the conditioning trials, rats had access to the entire cage, which provided a single tactile floor cue (either two mesh tiles or two bar tiles). On the day immediately after the final conditioning trial, a single $10 \mathrm{~min}$ test session was given. Here, the CPP cages contained one bar tile and one mesh tile. Animals in a drug-free state were placed in the middle of the cage and given free choice between the half of their cage with the bar texture and that with the mesh texture. Before a new test or conditioning session was started, half of the soiled Beta Chip was removed and replaced with new bedding, and the cage walls and tiles were wiped with $40 \%$ ethanol and allowed to dry. Groups of animals were counterbalanced as fully as possible, not only with respect to the texture that was paired with drug but also with respect to the position of that texture within the test cage on test day and the order of drug versus saline administration during conditioning.

On the test day, the time spent on each side of the apparatus was recorded. The location of a rat was defined as its center, as determined by the tracking system. During conditioning trials, locomotor activity was recorded as total horizontal distance moved. All testing was done between 8:30 A.M. and 5:30 P.M. A pilot study in which rats received saline paired with both floor textures showed that rats had no significant preference for either texture on test day (our unpublished observations). Thus the procedure can be considered unbiased.

Experimental procedures. In experiment 1, rats received bilateral infusion of 6-OHDA or vehicle into either core or medial shell 7-11 d before preexposure. Rats were then conditioned with $0.75 \mathrm{mg} / \mathrm{kg}$ AMPH intraperitoneally. In experiment 2 , rats received bilateral 6-OHDA or vehicle infusions into medial shell. Half of the rats in each surgery group received $0.75 \mathrm{mg} / \mathrm{kg}$ AMPH intraperitoneally; the other half were conditioned with $10 \mathrm{mg} / \mathrm{kg}$ morphine intraperitoneally. Experiment 3 is similar in design to experiment 1 except that rats underwent stereotaxic infusion surgery after conditioning but before testing (Fig. 1).

\section{Quantitative [ ${ }^{125}$ I]RTI-55 autoradiography}

The extent of the 6-OHDA lesion was quantified by autoradiographic labeling of the plasmalemmal DA transporter (DAT) using a nonsaturating concentration of $\left[{ }^{125} \mathrm{I}\right] \mathrm{RTI}-55(2200 \mathrm{Ci} / \mathrm{mmol}$; NEN-Mandel, Guelph, Ontario), because it has been shown previously that percentage loss of DAT accurately represents tissue DA loss (Joyce, 1991a,b). $\left[{ }^{125} \mathrm{I}\right] \mathrm{RTI}-55$ binds selectively to DAT provided the serotonin transporter (SERT) is inhibited (Boja et al., 1992; Coulter et al., 1995). Conversely, SERT can be selectively labeled via occlusion of DAT (Pradhan et al., 2002). The day after CPP testing, rats were anesthetized with sodium pentobarbital (65 mg/kg, i.p.) and decapitated. Brains were removed rapidly and frozen in 2-methylbutane at $-50^{\circ} \mathrm{C}$ for $30 \mathrm{sec}$ and stored at $-40^{\circ} \mathrm{C}$. Coronal sections $(20 \mu \mathrm{m})$ were taken on a cryostat at four rostrocaudal levels through the nucleus accumbens: $+11.2,+10.7,+10.2$, and $+9.7 \mathrm{~mm}$ anterior to interaural zero (Paxinos and Watson, 1997). At each level, five adjacent sections were collected: four for autoradiography and one for Nissl staining with cresyl violet. Sections were thaw mounted onto gelatin-subbed slides, air dried at room temperature for 20-30 min, and stored with desiccant at $-40^{\circ} \mathrm{C}$.

Sections were thawed at room temperature for $10 \mathrm{~min}$ and then placed in a staining dish containing an aqueous buffer solution of $120 \mathrm{~mm} \mathrm{NaCl}$, $0.1 \mathrm{~m}$ sucrose, $10 \mathrm{~mm}$ sodium phosphate buffer, and $10 \mathrm{pm}\left[{ }^{125} \mathrm{I}\right] \mathrm{RTI}-55$. To assay for DAT binding, $50 \mathrm{~nm}$ citalopram hydrobromide was used to occlude SERT; nonspecific binding was determined by addition of $10 \mu \mathrm{M}$ GBR 12909. To measure SERT binding, $1 \mu \mathrm{M}$ GBR $12935 \cdot 2 \mathrm{HCl}$ was added to occlude DAT; nonspecific binding was determined by addition of $50 \mathrm{~nm}$ citalopram $\mathrm{HBr}$ (Pradhan et al., 2002). Slides were incubated at room temperature for $2 \mathrm{hr}$ and then washed three times in cold buffer 


\section{Pre-

exposure (1d) Conditioning (6d) Test (1d)
$0.75 \mathrm{mg} / \mathrm{kg} \mathrm{AMPH} \mathrm{IP}(\mathrm{n}=46)$ \\ Experiment 1}

$0.75 \mathrm{mg} / \mathrm{kg}$ AMPH IP $(\mathrm{n}=22)$

$10 \mathrm{mg} / \mathrm{kg}$ morphine IP $(\mathrm{n}=22)$

\section{Experiment 2}

\section{$0.75 \mathrm{mg} / \mathrm{kg}$ AMPH IP $(\mathrm{n}=52)$}

\section{Experiment 3}

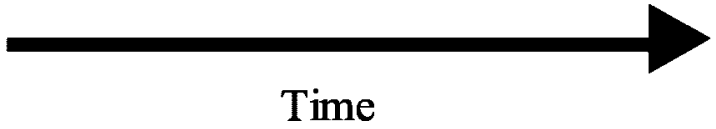

Figure 1. Experimental design of experiments 1,2 and 3 . Vehicle or 6-OHDA infusions were given at the time indicated by the arrows. In experiments 1 and 3, rats received infusions into either core or medial shell, depending on group (filled arrows). In experiment 2, only medial shell was targeted (white arrow). During the conditioning phase, each rat received saline and a drug (AMPH or morphine, dose as indicated) on alternating days (see Materials and Methods). IP, Intraperitoneal.

solution (once for $1 \mathrm{~min}$, twice for $20 \mathrm{~min}$ ) and for $1 \mathrm{sec}$ in distilled and deionized water. They were then blow dried and placed in an $\mathrm{x}$-ray film cassette. Kodak BioMax MS film (Amersham Biosciences, Baie d'Urfé, Québec) was exposed to slides for $48 \mathrm{hr}$ (DAT) or $120 \mathrm{hr}$ (SERT) with $\left[{ }^{125} \mathrm{I}\right]$ autoradiographic standards (Amersham Biosciences). Films were then processed with Kodak D19 developer and Kodak GBX fixer (Amersham Biosciences). DAT and SERT binding were quantified using an MCID M4 imaging system (Imaging Research, St. Catherines, Ontario).

\section{Histological examination}

Tissue was stained with cresyl violet to assess nonspecific damage, as follows. Sections were thawed at room temperature for $10 \mathrm{~min}$ and then placed in $0.5 \%$ cresyl violet (Sigma-Aldrich, Oakville, Ontario) in distilled water for $20 \mathrm{~min}$. They were rinsed in $95 \%$ ethanol twice for $2 \mathrm{~min}$ and then in $100 \%$ ethanol three times for $15 \mathrm{sec}$ and were dehydrated in xylene three times for $5 \mathrm{~min}$. Slides were coverslipped with Permount and examined under a light microscope (40-200× magnification).

\section{Drugs}

Drug sources were as follows: morphine sulfate (gift from Sabex 2002 Inc., Boucherville, Quebec); D-amphetamine sulfate (Bureau of Drug Research, Ottawa, Ontario); citalopram $\mathrm{HBr}$ (gift from H. Lundbeck A/S); dipyrone (Vetoquinol, Quebec, Quebec); ketamine $\mathrm{HCl}$ (Vetalar, Vetrepharm, London, Ontario); xylazine HCl (Anased, Novopharm, Toronto, Ontario); atropine methyl nitrate, 6-OHDA HBr, GBR 12909, and GBR $12935 \cdot 2 \mathrm{HCl}$ (Sigma-Aldrich, Oakville, Ontario). All other chemicals were obtained from Fisher Scientific (Montreal, Quebec).

Morphine sulfate and D-amphetamine sulfate were dissolved in sterile $0.9 \%$ saline and injected at $1 \mathrm{ml} / \mathrm{kg}$. 6-OHDA $\mathrm{HBr}$ was dissolved in sterile $0.9 \%$ saline containing $0.3 \mathrm{mg} / \mathrm{ml}$ sodium metabisulfite (Sigma-Aldrich) as an antioxidant and protected from light. Both 6-OHDA and vehicle solutions were made to $\mathrm{pH} 7.3 \pm 0.1$ with $\mathrm{NaOH}$. Doses of all drugs except 6-OHDA $\mathrm{HBr}$ are expressed as the salt. 6-OHDA $\mathrm{HBr}$ doses are expressed as free base.
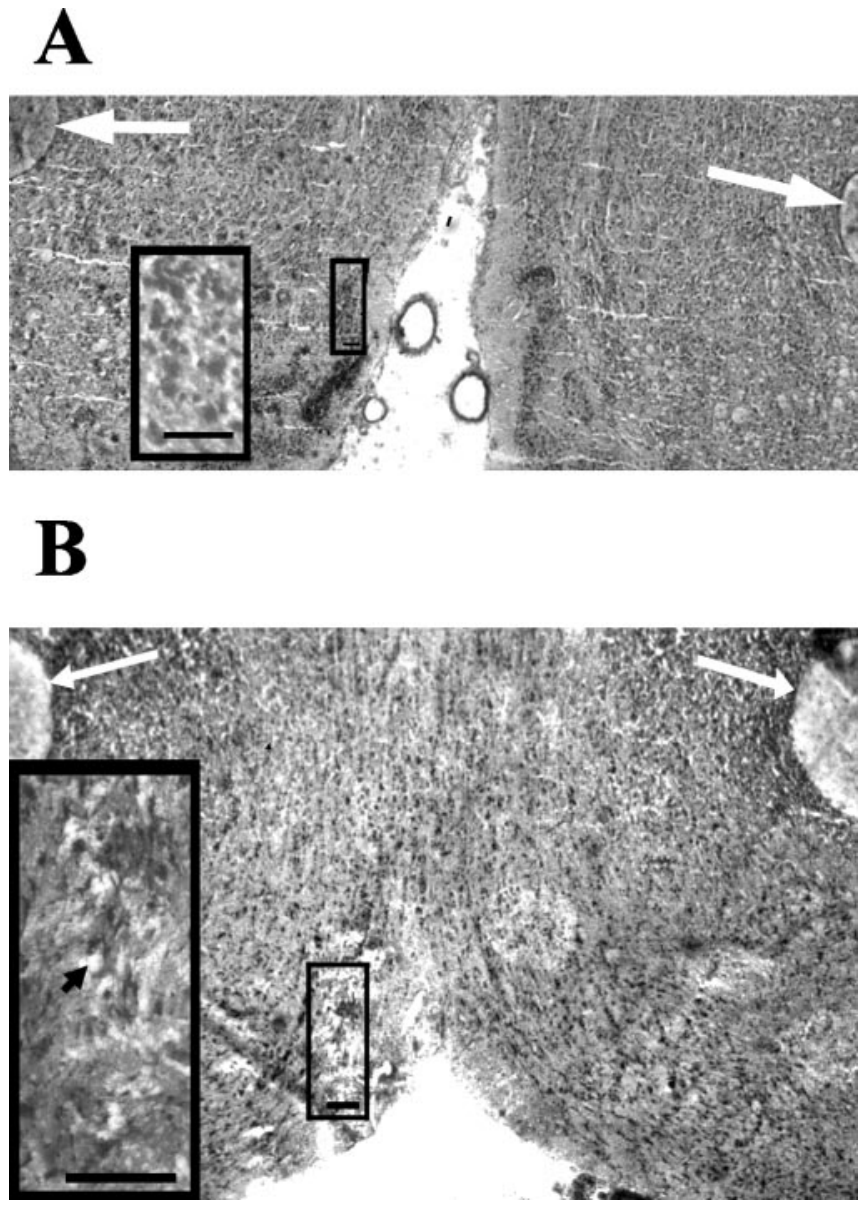

Figure 2. Histological changes associated with infusion of vehicle $(A)$ or 6-OHDA ( $B)$ into the medial shell region of the NAcc. Representative $20 \mu \mathrm{m}$ Nissl-stained sections are shown $\sim 0.1$ $\mathrm{mm}$ caudal to the site of injection (10.2 $\mathrm{mm}$ anterior to interaural zero). 6-0HDA infusion resulted in disruption of normal tissue morphology local to the infusion site ( $B$, black arrow). Much less disruption of normal tissue morphology occurred in rats infused with vehicle. Scale bars, $50 \mu \mathrm{m}$. Anterior commissure is indicated by white arrows.

\section{Data analysis}

A commercial software program (Systat v10.2, SPSS Inc., Chicago, IL) was used for all data analyses. Locomotor response to AMPH was calculated as the difference of locomotor counts between AMPH and saline conditioning sessions; baseline saline scores were calculated as the mean activity over all three conditioning sessions with saline on test day. CPP magnitude was calculated as the difference between time spent on the drug-paired and vehicle-paired sides. The relationship between locomotor and reward measures versus [ $\left.{ }^{125} \mathrm{I}\right]-\mathrm{RTI}-55$ labeling was analyzed by multiple linear regression (experiments 1 and 3) or Mann-Whitney $U$ test (experiment 2). Activity scores (experiment 1 ) were analyzed by ANOVA. A $p$ value of $<0.05$ (two-tailed) was considered significant.

\section{Results}

Histological and autoradiographic characterization of lesions Minimal neuronal loss was evident at the site of injection in both vehicle groups and in the core lesioned group in all three experiments. A representative coronal section of the medial shell vehicle-infused group is shown in Figure $2 \mathrm{~A}$. In the medial shell lesioned group, tissue damage was more extensive but was nevertheless confined to $0.3 \mathrm{~mm}$ from the infusion site, sparing most of the structure (Fig. $2 B$ ).

$\left[{ }^{125} \mathrm{I}\right]$ RTI-55 autoradiographs of DAT binding are shown in Figure 3 at four anterior-posterior levels. Sampling locations for DAT binding density are indicated in Figure 4. Absolute values 
for $\left[{ }^{125} \mathrm{I}\right] \mathrm{RTI}-55$ binding to DAT and SERT are given in Tables 1 and 2. In all experiments, core lesions were less anatomically selective than shell lesions (Fig. 5). Pooled across experiments, core 6-OHDA animals showed a mean decrease in DAT binding of $68 \%$ in core, $29 \%$ in medial shell, $30 \%$ in ventral shell, $37 \%$ in ventral caudate-putamen, and $30 \%$ in olfactory tubercle (OT). In contrast, medial shell-infused 6-OHDA reduced DAT binding in medial shell by $62 \%$, but only by $13,7,1$, and $12 \%$ in core, ventral shell, ventral caudate-putamen, and OT, respectively. SERT binding was virtually unchanged $(89-111 \%$ of control) by the 6-OHDA lesions in all three experiments (Tables 1, 2).

\section{NAcc core and medial shell lesions before conditioning inhibited AMPH- mediated locomotor activation and CPP, respectively}

In experiment 1 , lesions were performed before drug conditioning. Overall, the AMPH locomotor stimulant effect differed across successive conditioning sessions (SESSION: $F_{(2,84)}=4.47, p<0.02$; mean \pm SEM; AMPH-saline difference score $40 \pm 5,63 \pm 6$, and $53 \pm 8 \mathrm{~m})$. However, locomotor data were pooled across sessions, because an initial three-way ANOVA revealed no significant interactions between SESSION and either AREA or LESION $\left(F_{(2,84)}<1.31, p>0.2\right)$. Saline session locomotor scores did not differ significantly between surgery groups $\left(\right.$ AREA: $F_{(1,42)}=1.01, p>0.25 ;$ LESION: $F_{(1,42)}=0.70, p>0.25$; AREA $\times$ LESION: $\left.F_{(1,42)}=0.95, p>0.25\right)$ (Fig. 6, legend). Because lesions were not anatomically specific (Fig. 4), multiple linear regression analysis was performed to assess contributions of core and shell DA innervation to the AMPH-induced locomotor response. Figure $6, A$ and $B$, shows the relationship between locomotor responses to AMPH during conditioning versus DAT binding in core and medial shell. The locomotor stimulant response was significantly correlated with DAT binding in NAcc core $(p<0.01)$ but not NAcc medial shell $(p>0.25)$ (Fig. $6 A, B)$. Conversely, the magnitude of AMPH CPP was significantly correlated with residual DAT in the medial shell $(p<$ $0.0001)$ but not in the core $(p>0.5)$ (Fig. 6C,D).

\section{NAcc medial shell lesions did not prevent acquisition of a CPP for morphine}

In experiment 2, the effects of preconditioning lesions of medial shell were tested in rats conditioned with either morphine (10 $\mathrm{mg} / \mathrm{kg}$, i.p.) or AMPH $(0.75 \mathrm{mg} / \mathrm{kg}$, i.p.). As in experiment 1 , AMPH CPP magnitude was reduced or abolished by medial shell 6-OHDA infusion (lesion vs sham: Mann-Whitney $U=90 ; p<$ 0.02) (Fig. 7). In contrast, lesioned rats did acquire a morphine $\mathrm{CPP}$, and this was of similar magnitude to that of sham controls (lesion vs sham: Mann-Whitney $U=63 ; p>0.5$ ) (Fig. 7).

Expression of a conditioned place preference for AMPH was abolished by NAcc medial shell, but not NAcc core, lesions In experiment 3 , lesions were performed after conditioning but before testing. Figure $8, A$ and $B$, shows the relationship between

\section{Core Sham Medial Shell}

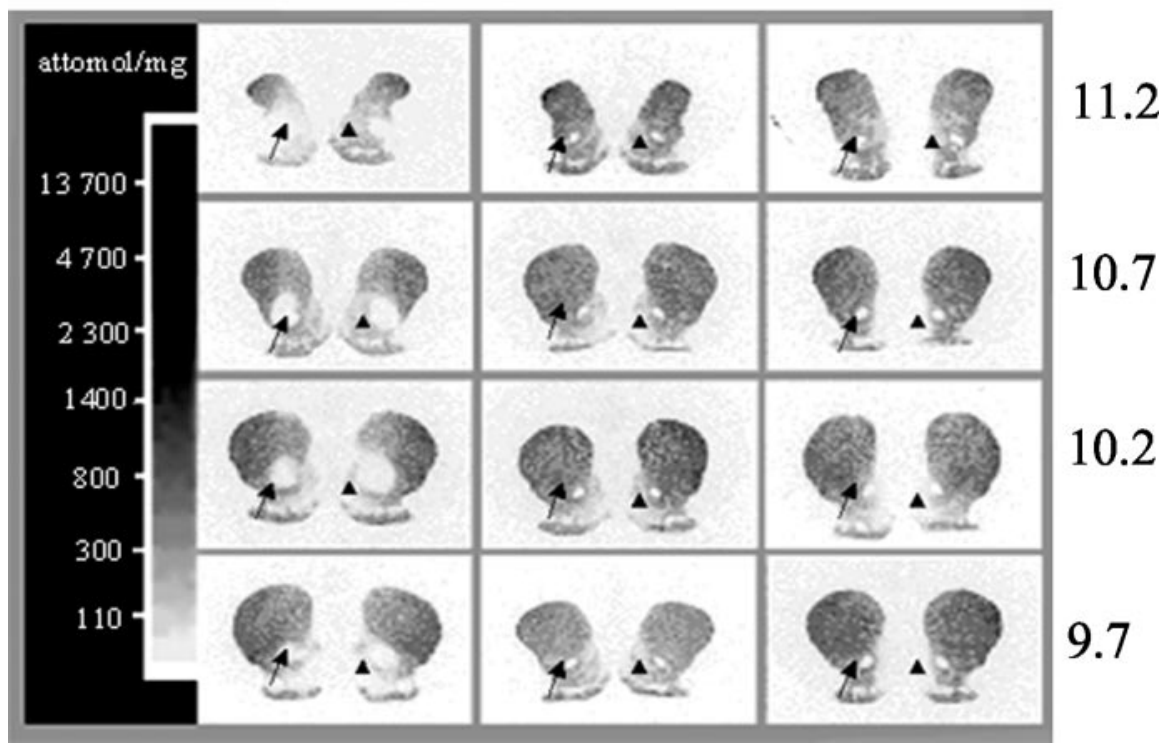

11.2

10.7 0.2

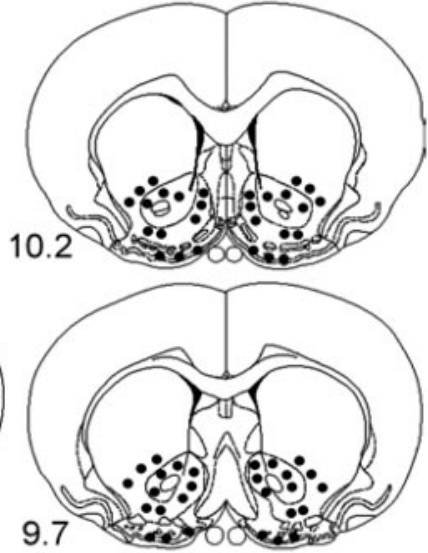

9.7

Figure 4. Locations of sampled [ $\left.{ }^{125} \mathrm{I}\right] \mathrm{RT}$ T-55 binding in nucleus accumbens core, medial shell, ventral shell, ventral caudate-putamen, and olfactory tubercle. Each rat was sampled at four anterior-posterior levels. Numbers are distances (in millimeters) anterior to interaural zero. Sampling areas were circles of $0.3 \mathrm{~mm}$ diameter. Three samples per side per structure were taken at each level, except for ventral shell, where one sample per side was taken at level 11.2 and two per side at all other levels. Adapted from Paxinos and Watson (1997).

DAT binding in NAcc core or medial shell and the CPP magnitude. Two extreme outliers, as defined by the Systat software, were excluded before data analysis. Multiple linear regression analysis showed that CPP magnitude correlated significantly with residual DAT binding in NAcc medial shell $(p<0.0005)$ but not in NAcc core $(p>0.25)$.

\section{Discussion}

\section{Methodological aspects}

Dopaminergic denervation in core or medial shell has rarely been achieved with any anatomical selectivity (Boye et al., 2001). The present study incorporated several methodological improve- 
Table 1. Absolute values of DAT and SERT binding in core, medial shell, ventral shell, ventral caudate-putamen (ventral CP), and olfactory tubercle (OT) in experiments 1 and 3

\begin{tabular}{|c|c|c|c|c|}
\hline & Core (vehicle) & Core (6-OHDA) & Shell (vehicle) & Shell (6-OHDA) \\
\hline \multicolumn{5}{|l|}{ Experiment 1} \\
\hline$n$ & 10 & 12 & 10 & 14 \\
\hline \multicolumn{5}{|l|}{ DAT } \\
\hline Core & $265 \pm 20$ & $87 \pm 9$ & $261 \pm 30$ & $209 \pm 20$ \\
\hline Medial shell & $183 \pm 7$ & $128 \pm 13$ & $178 \pm 18$ & $70 \pm 20$ \\
\hline Ventral shell & $170 \pm 8$ & $129 \pm 17$ & $158 \pm 15$ & $166 \pm 16$ \\
\hline Ventral CP & $152 \pm 9$ & $95 \pm 11$ & $129 \pm 11$ & $137 \pm 7$ \\
\hline OT & $259 \pm 16$ & $177 \pm 18$ & $282 \pm 24$ & $243 \pm 16$ \\
\hline \multicolumn{5}{|l|}{ SERT } \\
\hline Core & $117 \pm 12$ & $107 \pm 14$ & $117 \pm 8$ & $113 \pm 7$ \\
\hline Medial shell & $145 \pm 14$ & $149 \pm 14$ & $143 \pm 6$ & $137 \pm 10$ \\
\hline Ventral shell & $151 \pm 17$ & $167 \pm 16$ & $136 \pm 14$ & $147 \pm 15$ \\
\hline Ventral CP & $152 \pm 17$ & $165 \pm 16$ & $141 \pm 17$ & $143 \pm 12$ \\
\hline OT & $219 \pm 9$ & $230 \pm 11$ & $242 \pm 13$ & $232 \pm 9$ \\
\hline \multicolumn{5}{|l|}{ Experiment 3} \\
\hline$n$ & 10 & 13 & 10 & 19 \\
\hline \multicolumn{5}{|l|}{ DAT } \\
\hline Core & $927 \pm 43$ & $288 \pm 60$ & $941 \pm 32$ & $816 \pm 61$ \\
\hline Medial shell & $566 \pm 45$ & $411 \pm 41$ & $596 \pm 56$ & $225 \pm 26$ \\
\hline Ventral shell & $774 \pm 38$ & $493 \pm 64$ & $787 \pm 40$ & $761 \pm 40$ \\
\hline Ventral CP & $641 \pm 31$ & $404 \pm 35$ & $684 \pm 19$ & $660 \pm 30$ \\
\hline OT & $733 \pm 36$ & $524 \pm 45$ & $743 \pm 53$ & $635 \pm 36$ \\
\hline \multicolumn{5}{|l|}{ SERT } \\
\hline Core & $297 \pm 10$ & $269 \pm 16$ & $310 \pm 16$ & $318 \pm 14$ \\
\hline Medial shell & $450 \pm 15$ & $441 \pm 16$ & $450 \pm 23$ & $446 \pm 14$ \\
\hline Ventral shell & $476 \pm 27$ & $486 \pm 23$ & $497 \pm 19$ & $504 \pm 20$ \\
\hline Ventral CP & $300 \pm 13$ & $267 \pm 10$ & $305 \pm 12$ & $321 \pm 12$ \\
\hline OT & $637 \pm 25$ & $624 \pm 35$ & $637 \pm 24$ & $664 \pm 26$ \\
\hline
\end{tabular}

Values are mean \pm SEM $\left[{ }^{125}\right.$ I]RTI-55 binding to DAT or SERT (expressed as attomol per milligram of tissue), obtained at a subsaturating concentration of radioligand.

Table 2. Absolute values of DAT and SERT binding in core, medial shell, ventral shell, ventral caudate-putamen (ventral CP), and olfactory tubercle (OT) in experiment 2

\begin{tabular}{|c|c|c|c|c|}
\hline & \multicolumn{2}{|l|}{ Morphine } & \multicolumn{2}{|l|}{ Amphetamine } \\
\hline & Shell (vehicle) & Shell (6-OHDA) & Shell (vehicle) & Shell (6-0HDA) \\
\hline \multicolumn{5}{|l|}{ Experiment 2} \\
\hline$n$ & 10 & 12 & 10 & 12 \\
\hline \multicolumn{5}{|l|}{ DAT } \\
\hline Core & $755 \pm 22$ & $715 \pm 20$ & $760 \pm 25$ & $674 \pm 20$ \\
\hline Medial shell & $473 \pm 17$ & $166 \pm 21$ & $407 \pm 53$ & $157 \pm 17$ \\
\hline Ventral shell & $429 \pm 12$ & $382 \pm 14$ & $437 \pm 21$ & $364 \pm 15$ \\
\hline Ventral CP & $535 \pm 18$ & $525 \pm 9$ & $517 \pm 21$ & $501 \pm 16$ \\
\hline OT & $670 \pm 18$ & $612 \pm 32$ & $695 \pm 43$ & $618 \pm 34$ \\
\hline \multicolumn{5}{|l|}{ SERT } \\
\hline Core & $414 \pm 14$ & $408 \pm 14$ & $385 \pm 9$ & $382 \pm 15$ \\
\hline Medial shell & $517 \pm 14$ & $530 \pm 19$ & $510 \pm 12$ & $501 \pm 23$ \\
\hline Ventral shell & $481 \pm 43$ & $534 \pm 50$ & $533 \pm 55$ & $474 \pm 41$ \\
\hline Ventral CP & $327 \pm 31$ & $343 \pm 29$ & $354 \pm 40$ & $322 \pm 26$ \\
\hline OT & $737 \pm 24$ & $688 \pm 18$ & $672 \pm 19$ & $672 \pm 24$ \\
\hline
\end{tabular}

Values are mean \pm SEM [ ${ }^{125}$ I]RTI-55 binding to DAT or SERT (expressed as attomol per milligram of tissue), obtained at a subsaturating concentration of radioligand.

ments. First, stereotaxic lesion coordinates were improved. Second, multiple infusion sites were used for medial shell lesions. Third, diffusion of 6-OHDA from the infusion site was minimized by administering a high concentration in a small volume. Consequently, core and medial shell DAT binding were largely independent (Pearson $r=0.30$ ), which was not the case in our previous study (Pearson $r=0.84$ ) (Boye et al., 2001). In addition, nonspecific tissue damage was reduced by neutralizing the 6-OHDA solution before infusion. Thus, despite the unusually high concentration of 6-OHDA used, Nissl staining and SERT autoradiography revealed minimal nonspecific damage.

The present CPP procedure possesses several attractive features. First, latent inhibition can be avoided during the initial preexposure phase by omitting the conditioned stimuli. Second, rats conditioned with saline on both textures showed no significant preference for either texture on test day (our unpublished observations). Hence, our procedure is balanced and avoids the interpretational difficulties inherent in "biased" procedures (Bardo and Bevins, 2000). The current study is the first to show an AMPH CPP using solely tactile cues.

Mechanisms of amphetamine-induced locomotor activation The present findings suggest that after systemic AMPH administration, locomotor stimulation is dependent on transmission in NAcc core and not medial shell. To date, only three published studies have examined this question using systemic rather than 


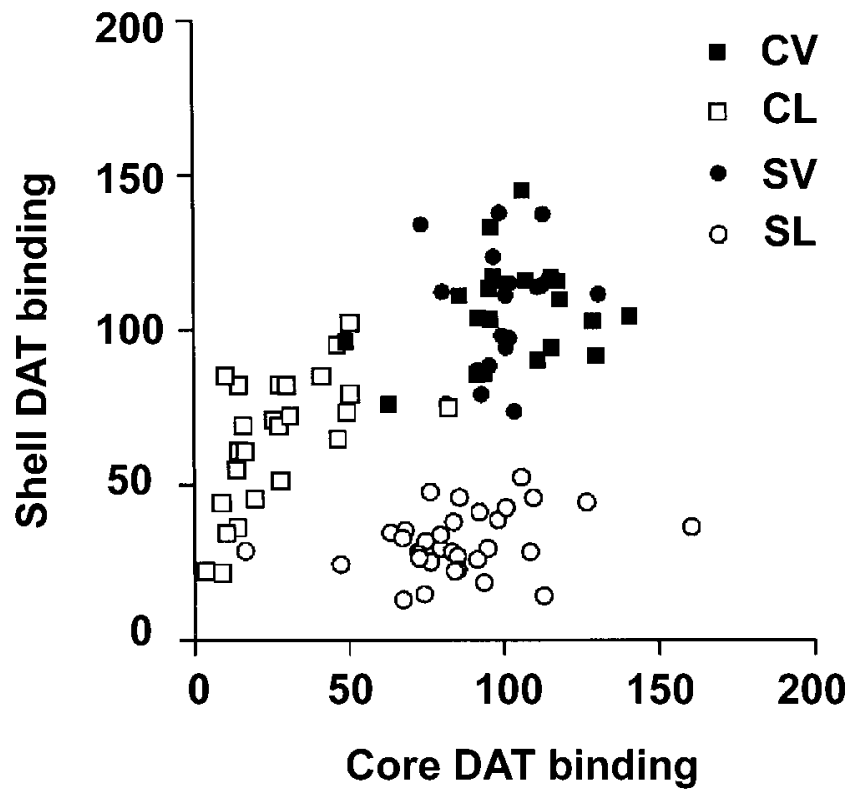

Figure 5. Relationship of DAT labeling in nucleus accumbens core versus medial shell. Data are pooled from experiments 1 and 3 ( $n=98$ rats). DAT labeling was performed by [ ${ }^{125}$ I]RTI-55 autoradiography and expressed as a percentage of the mean value of the core-vehicle group for core 6-OHDA animals, or the shell-vehicle group for the shell 6-0HDA group. Correlational analysis revealed a weak but significant relationship between core and medial shell binding $(r=0.30 ; p<0.005)$. CV, Core vehicle; CL, core lesioned; SV, medial shell vehicle; SL, medial shell lesion.

intracranial AMPH (Weiner et al., 1996; Parkinson et al., 1999; Boye et al., 2001). Two of these studies showed that core rather than shell lesions reduced AMPH-induced locomotor activation (Weiner et al., 1996; Boye et al., 2001). In contrast, Parkinson et al. (1999) reported that excitotoxic lesions of the NAcc core enhanced locomotor stimulant responses to systemic AMPH, whereas medial shell lesions had the opposite effect. On this basis, these authors attributed a critical role to the shell; however, in the latter study, shell lesions attenuated AMPH locomotion to only a modest extent, and core lesions increased baseline locomotion, complicating the interpretation of drug effects. On balance, therefore, the available evidence suggests that NAcc core plays an important role in the locomotor stimulant effect of systemically administered AMPH.

In the present study, medial shell DA innervation was not related to AMPH locomotor stimulation. In contrast, we previously observed a significant negative correlation $(p<0.02)$, such that DA denervation in the medial shell was associated with greater locomotor responses (Boye et al., 2001). This discrepancy may reflect differences in lesions coordinates or functional gradients within each NAcc subregion (Essman et al., 1993; Campbell et al., 1997).

Other striatal regions, notably ventromedial striatum (Dickson et al., 1994), OT (Cools, 1986; Ikemoto, 2002), and anteromedial caudate (Fink and Smith, 1979, 1980), have also been implicated in AMPH-induced locomotion. It is doubtful that denervation of ventromedial striatum played a significant role in the present study, because lesions were restricted to the anterior portion, which appears not to contribute to AMPH locomotor activation (Dickson et al., 1994). On the basis of intracranial infusion studies (Cools, 1986; Ikemoto, 2002), the OT has been proposed as a key structure mediating the locomotor stimulant effects of AMPH. In contrast, locomotor stimulation after sys-
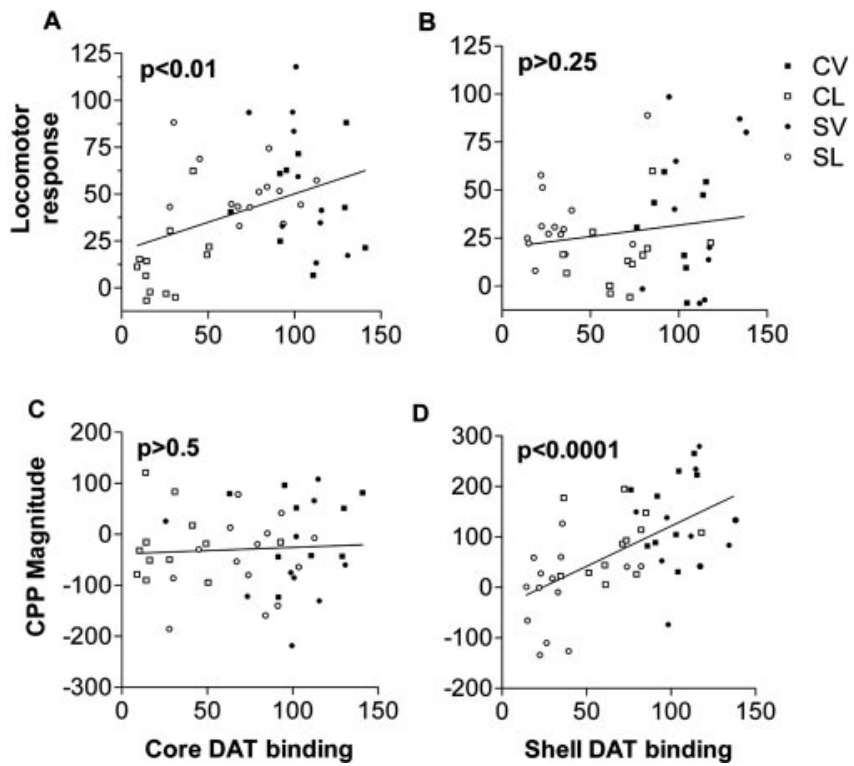

Figure 6. Effect of bilateral 6-OHDA infusion into either NAcc core or medial shell on AMPHinduced locomotor response and CPP (experiment 1). Rats ( $n=10-14$ per group) were allowed 7-11 d recovery after stereotaxic surgery before conditioning with AMPH $(0.75 \mathrm{mg} / \mathrm{kg}$, i.p.). Locomotor responses are expressed for each rat as the difference between the mean distance moved (in meters) during conditioning sessions with AMPH versus with saline. CPP magnitude is the difference between time spent on the drug-paired and saline-paired textures during the 600 sec test. DAT labeling in core or medial shell is expressed as percentage DAT binding of sham-lesioned groups. Saline locomotor scores, in meters, were $134 \pm 7$ in the core vehicle group, $152 \pm 11$ in the core $6-$ OHDA group, $154 \pm 10$ in the shell vehicle group, and $153 \pm 11$ in the shell 6-OHDA group. Locomotor responses (AMPH-saline) correlated significantly with DAT binding in NAcc core but not in NAcc medial shell. Conversely, CPP magnitude correlated significantly with DAT binding in medial shell but not core. To visualize the association of each drug response to core or medial shell [ ${ }^{125}$ I]RTI-55 labeling, the predicted contribution of the irrelevant brain structure was subtracted from the $y$-axis variables using the calculated multiple linear regression equation. Significant linear associations (shown by $p$ values) are evident in $A$ and $D$. CV, Core vehicle; CL, core lesioned; SV, medial shell vehicle; $S L$, medial shell lesion.

temic AMPH administration was unaffected by 6-OHDA lesions of OT, despite substantial loss of tissue DA (Clarke et al., 1988). The anteromedial caudate has been proposed to mediate AMPHinduced locomotion (Fink and Smith, 1979), but this area was probably spared by our lesions. The ventral shell subregion was partially depleted by our core 6-OHDA infusions and, to our knowledge, has not been studied with respect to AMPH locomotion.

Our 6-OHDA infusions almost certainly destroyed noradrenaline (NA) as well as DA terminals in the ventral striatum (Robbins et al., 1983). Disruption of noradrenergic transmission tends to inhibit AMPH-induced locomotion (Ogren et al., 1983; Archer et al., 1986; Dickinson et al., 1988; Blanc et al., 1994; Darracq et al., 1998; Harro et al., 2000; Drouin et al., 2002a,b; Auclair et al., 2002) [but see Ventura et al. (2003)], with the medial prefrontal cortex identified as a potential site of action (Blanc et al., 1994; Darracq et al., 1998). In contrast, noradrenergic transmission in the NAcc appears not to contribute directly to locomotor stimulation (Pijnenburg et al., 1975; Roberts et al., 1975; Kelly and Iversen, 1976; Joyce et al., 1983). Thus, our 6-OHDA lesion effects on AMPH-induced locomotor activation probably reflect decreased DA rather than NA transmission.

\section{Mechanisms of AMPH-induced reward}

Considerable evidence suggests that AMPH exerts its rewarding effects via DA release in the NAcc (Di Chiara, 1995; Koob et al., 


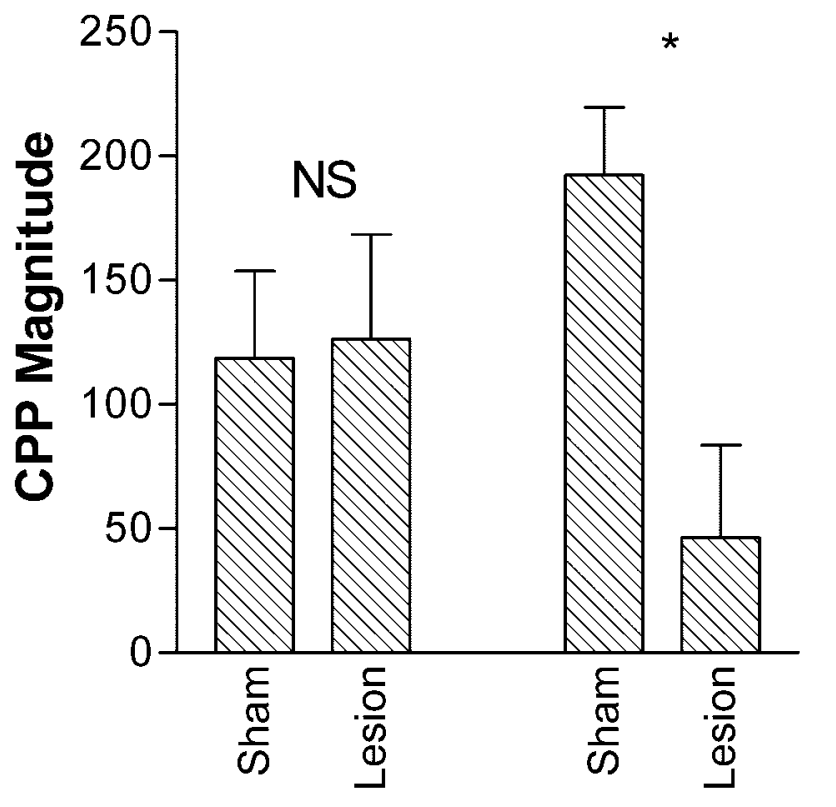

Morphine Amphetamine

Figure 7. Effect of 6-OHDA lesions of NAcc medial shell on morphine and AMPH CPP (experiment 2). Stereotaxic surgery was performed $7-11 \mathrm{~d}$ before the first conditioning day. (PP magnitudes (mean \pm SEM) for morphine $(10 \mathrm{mg} / \mathrm{kg}$, i.p.) or AMPH $(0.75 \mathrm{mg} / \mathrm{kg}$, i.p.) were calculated as the difference between the time spent on the drug-paired and saline-paired sides ( $n=10-12$ rats per group). Because the data were not normally distributed, Mann-Whitney $U$ tests were applied to predetermined comparisons. NS, Nonsignificant; ${ }^{*} p<0.02$ versus corresponding sham-lesioned group (unprotected tests).

1998), with little if any contribution from NA in this structure (Yokel and Wise, 1975; Roberts et al., 1977). In the present study, medial shell DA denervation was associated with attenuated AMPH CPP. It is unlikely that neighboring structures contributed to this effect, because they were only slightly denervated (Tables 1, 2). Moreover, substantial 6-OHDA lesions of OT did not alter a CPP for systemic AMPH (Clarke et al., 1990). Our findings therefore support a role for NAcc medial shell DA in the rewarding effect of AMPH. This conclusion accords with intracranial self-administration studies using other dopaminergic drugs (Carlezon and Wise, 1996; Ikemoto et al., 1997).

The inhibition of AMPH CPP caused by preconditioning 6-OHDA lesions could reflect impaired acquisition or expression, or both. It is well established that acquisition and expression of CPP are mediated by different dopaminergic mechanisms (Hiroi and White, 1990, 1991a,b; Acquas and Di Chiara, 1994; Bardo et al., 1999). For example, DA antagonist studies show that DA D1 and D2 receptors are required for acquisition, but only DA D1 receptors are required for expression (Hiroi and White, 1991a,b; Acquas and Di Chiara, 1994; Bardo et al., 1999). Because our 6-OHDA lesions presumably impaired transmission at both DA receptor types, both acquisition and expression are likely to be affected.

In the present study, morphine served as a positive control. The finding that morphine CPP was unaffected by medial shell lesions (experiment 2) suggests that lesion-induced reduction of AMPH CPP did not result from impaired sensory, motor, or mnemonic function. The present findings also accord with evidence that morphine CPP occurs via a DA-independent mechanism when drug exposure is minimized (Mackey and van der Kooy, 1985; Bechara and van der Kooy, 1992; Bechara et al., 1992;

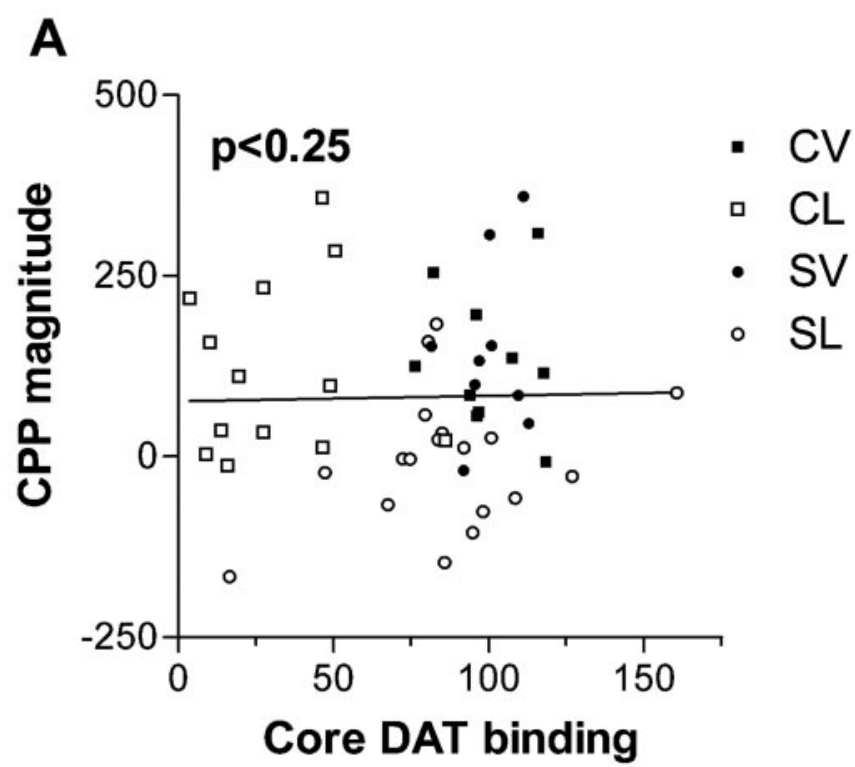

B

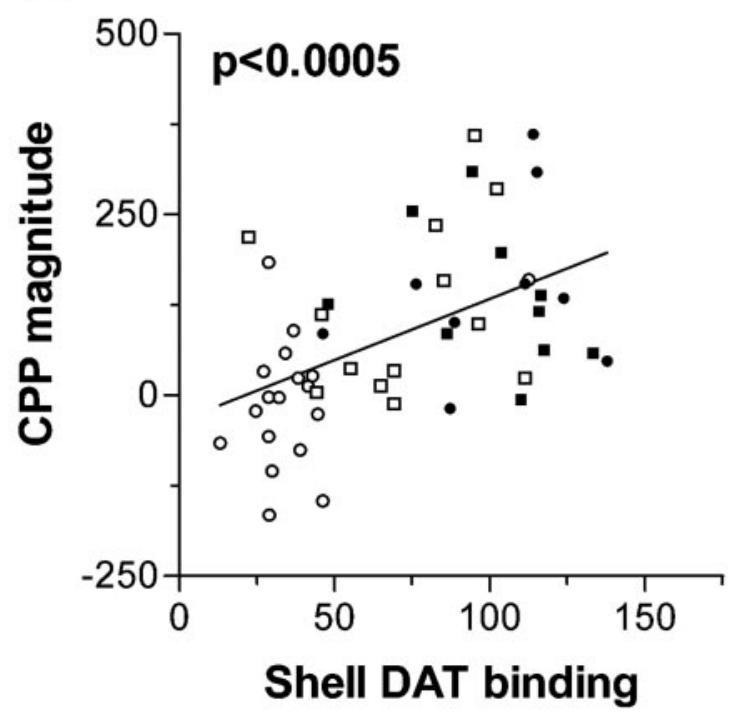

Figure 8. Effect of NAcc core and medial shell lesions on the expression of AMPH CPP (experiment 3). Rats ( $n=10-19$ per group) received bilateral infusion of either $6-0 H D A$ or vehicle into either NAcc core or medial shell after conditioning with AMPH and before CPP testing. Degree of DAT depletion in core or medial shell is expressed as percentage DAT binding of control. To visualize the association of each drug response to core or medial shell [ ${ }^{125}$ ] $]$ RTI-55 labeling, the predicted contribution of the irrelevant brain structure was subtracted from the $y$-axis variables using the calculated multiple linear regression equation. (PP magnitude correlated significantly with DAT binding in NAcc medial shell $(B)$ but not with DAT binding in NAcc core $(A)$. CV, Core vehicle; $\mathrm{CL}$, core lesioned; SV, medial shell vehicle; $\mathrm{SL}$, medial shell lesion.

Nader and van der Kooy, 1997; Laviolette et al., 2002). In contrast, 6-OHDA lesions of the NAcc have been found to reduce opiate CPP in nondependent rats (Spyraki et al., 1983; Shippenberg et al., 1993). Several factors could account for this discrepancy. First, these authors denervated the entire NAcc. It is possible that the NAcc medial shell subregion is neither necessary nor sufficient to mediate opiate reward. Second, although our lesions eliminated AMPH CPP, they may not have decreased DA transmission sufficiently to affect morphine CPP. Third, it is possible that different neural mechanisms underlie morphine CPP depending on whether multiple sensory cues or solely tactile cues are used. 


\section{Dissociation of locomotion and reward}

The current findings demonstrate a double dissociation in NAcc core versus shell with regard to AMPH-induced locomotor activation and reward. They extend evidence from other behavioral paradigms that also suggest that locomotion and reward are dissociable (Burns et al., 1993; Robledo et al., 1993; Kelley et al., 1997; Ventura et al., 2003). Burns et al. (1993) performed lesions of the ventral subiculum or basolateral amygdala and demonstrated a double dissociation of the locomotor stimulation and conditioned reinforcement produced by intra-NAcc AMPH. However, it is not clear whether the lesion affected reward processes or produced a memory or sensory deficit. Robledo et al. (1993) showed that neurotensin administered into the NAcc core decreased the locomotor stimulant effect of cocaine but did not affect intravenous self-administration of the drug. In this study, cocaine was given intraperitoneally in the locomotor tests, making interpretation difficult. Kelley et al. (1997) found that administration of an NMDA receptor antagonist into NAcc core, but not shell, disrupted the acquisition of food-reinforced responding without affecting spontaneous locomotor activity. However, this study did not examine the effects of psychostimulants. Last, Ventura et al. (2003) demonstrated that in mice, NA-depleting lesions of the medial prefrontal cortex blocked both AMPHinduced NAcc DA release and CPP while preserving the locomotor stimulant response. It would be interesting to determine whether core and medial shell DA release are differentially affected by these lesions and whether this result extends to rats.

In conclusion, the present study provides the first clear anatomical dissociation between the rewarding and locomotoractivating effects of the prototypic psychostimulant drug AMPH in rats. These acute behavioral effects were mapped onto NAcc medial shell and core, respectively. The experimental approach used here should help to further define mechanisms underlying acute and chronic behavioral effects of other drugs of abuse. Finally, the present core/shell dissociation may be relevant to the role of DA in reward anticipation versus consumption (Wise, 2002), incentive salience (Berridge and Robinson, 1998), and other forms of learning (Redgrave et al., 1999; Schultz, 2002).

\section{References}

Acquas E, Di Chiara G (1994) D1 receptor blockade stereospecifically impairs the acquisition of drug-conditioned place preference and place aversion. Behav Pharmacol 5:555-569.

Archer T, Fredriksson A, Jonsson G, Lewander T, Mohammed AK, Ross SB, Soderberg U (1986) Central noradrenaline depletion antagonizes aspects of D-amphetamine-induced hyperactivity in the rat. Psychopharmacology 88:141-146.

Auclair A, Cotecchia S, Glowinski J, Tassin J-P (2002) D-Amphetamine fails to increase extracellular dopamine levels in mice lacking $\alpha$ lb-adrenergic receptors: relationship between functional and nonfunctional dopamine release. J Neurosci 22:9150-9154.

Bardo MT, Bevins RA (2000) Conditioned place preference: what does it add to our preclinical understanding of drug reward? Psychopharmacology 153:31-43.

Bardo MT, Valone JM, Bevins RA (1999) Locomotion and conditioned place preference produced by acute intravenous amphetamine: role of dopamine receptors and individual differences in amphetamine selfadministration. Psychopharmacology 143:39-46.

Bechara A, van der Kooy D (1992) A single brain stem substrate mediates the motivational effects of both opiates and food in nondeprived rats but not in deprived rats. Behav Neurosci 106:351-363.

Bechara A, Harrington F, Nader K, van der Kooy D (1992) Neurobiology of motivation: double-dissociation of two motivational mechanisms mediating opiate reward in drug-naive vs. drug-dependent animals. Behav Neurosci 106:798-807.

Berridge KC, Robinson TE (1998) What is the role of dopamine in reward: hedonic impact, reward learning or incentive salience? Brain Res Rev 28:309-369.

Blanc G, Trovero F, Vezina P, Herve D, Godeheu A-M, Glowinski J, Tassin J-P (1994) Blockade of prefronto-cortical alphal-adrenergic receptors prevents locomotor hyperactivity induced by subcortical D-amphetamine injection. Eur J Neurosci 6:293-298.

Boja JW, Mitchell WM, Patel A, Kopajtic TA, Carroll FI, Lewin AH, Abraham P, Kuhar MJ (1992) High-affinity binding of [ ${ }^{125}$ I]RTI-55 to dopamine and serotonin transporters in rat brain. Synapse 12:27-36.

Boye SM, Grant RJ, Clarke PBS (2001) Disruption of dopaminergic neurotransmission in nucleus accumbens core inhibits the locomotor stimulant effects of nicotine and D-amphetamine in rats. Neuropharmacology 40:792-805.

Burns LH, Robbins TW, Everitt BJ (1993) Differential effects of excitotoxic lesions of the basolateral amygdala, ventral subiculum and medial prefrontal cortex on responding with conditioned reinforcement and locomotor activity potentiated by intra-accumbens infusions of D-amphetamine. Behav Brain Res 55:167-183.

Campbell A, Villavicencio AT, Yeghiayan SK, Balikian R, Baldessarini RJ (1997) Mapping of locomotor behavioral arousal induced by microinjections of dopamine within nucleus accumbens septi of rat forebrain. Brain Res 771:55-62.

Carlezon WA, Wise RA (1996) Rewarding actions of phencyclidine and related drugs in nucleus accumbens shell and frontal cortex. J Neurosci 16:3112-3122.

Carr GD, White NM (1991) Conditioned place preference from intraaccumbens but not intra-caudate amphetamine injections. Life Sci 33:2551-2557.

Chevrette J, Stellar JR, Hesse GW, Markou A (2001) Both the shell of the nucleus accumbens and the central nucleus of the amygdala support amphetamine self-administration in rats. Pharmacol Biochem Behav 71:501-507.

Clarke PBS, Jakubovic A, Fibiger HC (1988) Anatomical analysis of the involvement of mesolimbocortical dopamine in the locomotor stimulant actions of D-amphetamine and apomorphine. Psychopharmacology 96:511-520.

Clarke PBS, White NM, Franklin KB (1990) 6-Hydroxydopamine lesions of the olfactory tubercle do not alter $(+)$-amphetamine-conditioned place preference. Behav Brain Res 36:185-188.

Cools AR (1986) Mesolimbic dopamine and its control of locomotor activity in rats: differences in pharmacology and light/dark periodicity between the olfactory tubercle and the nucleus accumbens. Psychopharmacology $88: 451-459$.

Coulter CL, Happe HK, Bergman DA, Murrin LC (1995) Localization and quantification of the dopamine transporter: comparison of $\left[{ }^{3} \mathrm{H}\right]$ WIN 35 , 428 and $\left[{ }^{125}\right.$ I] RTI-55. Brain Res 690:217-224.

Darracq L, Blanc G, Glowinski J, Tassin JP (1998) Importance of the noradrenaline-dopamine coupling in the locomotor activating effects of D-amphetamine. J Neurosci 18:2729-2739.

Di Chiara G (1995) The role of dopamine in drug abuse viewed from the perspective of its role in motivation. Drug Alcohol Depend 38:95-137.

Dickinson SL, Gadie B, Tulloch IF (1988) Alpha 1- and alpha 2 -adrenoreceptor antagonists differentially influence locomotor and stereotyped behaviour induced by D-amphetamine and apomorphine in the rat. Psychopharmacology 96:521-527.

Dickson PR, Lang CG, Hinton SC, Kelley AE (1994) Oral stereotypy induced by amphetamine microinjection into striatum: an anatomical mapping study. Neuroscience 61:81-91.

Drouin C, Darracq L, Trovero F, Blanc G, Glowinski J, Cotecchia S, Tassin JP (2002a) Alphalb-adrenergic receptors control locomotor and rewarding effects of psychostimulants and opiates. J Neurosci 22:2873-2884.

Drouin C, Blanc G, Villegier AS, Glowinski J, Tassin JP (2002b) Critical role of alpha1-adrenergic receptors in acute and sensitized locomotor effects of D-amphetamine, cocaine, and GBR 12783: influence of preexposure conditions and pharmacological characteristics. Synapse 43:51-61.

Essman WD, McGonigle P, Lucki I (1993) Anatomical differentiation within the nucleus accumbens of the locomotor stimulatory actions of selective dopamine agonists and D-amphetamine. Psychopharmacology 112:233-241.

Fink JS, Smith GP (1979) Abnormal pattern of amphetamine locomotion after 6-OHDA lesion of anteromedial caudate. Pharmacol Biochem Behav 11:23-30.

Fink JS, Smith GP (1980) Mesolimbicocortical dopamine terminal fields are 
necessary for normal locomotor and investigatory exploration in rats. Brain Res 199:359-384.

Harro J, Merikula A, Lepiku M, Modiri AR, Rinken A, Oreland L (2000) Lesioning of locus coeruleus projections by DSP-4 neurotoxin treatment: effect on amphetamine-induced hyperlocomotion and dopamine D2 receptor binding in rats. Pharmacol Toxicol 86:197-202.

Heidbreder C, Feldon J (1998) Amphetamine-induced neurochemical and locomotor responses are expressed differentially across the anteroposterior axis of the core and shell subterritories of the nucleus accumbens. Synapse 29:310-322.

Hiroi N, White NM (1990) The reserpine-sensitive dopamine pool mediates $(+)$-amphetamine-conditioned reward in the place preference paradigm. Brain Res 510:33-42.

Hiroi N, White NM (1991a) The lateral nucleus of the amygdala mediates expression of the amphetamine-produced conditioned place preference. J Neurosci 11:2107-2116.

Hiroi N, White NM (1991b) The amphetamine conditioned place preference: differential involvement of dopamine receptor subtypes and two dopaminergic terminal areas. Brain Res 552:141-152.

Hoebel BG, Monaco AP, Hernandez L, Aulisi EF, Stanley BG, Lenard L (1983) Self-injection of amphetamine directly into the brain. Psychopharmacology 81:158-163.

Ikemoto S (2002) Ventral striatal anatomy of locomotor activity induced by cocaine, D-amphetamine, dopamine and D1/D2 agonists. Neuroscience 113:939-955.

Ikemoto S, Glazier BS, Murphy JM, McBride WJ (1997) Role of dopamine $\mathrm{D} 1$ and D2 receptors in the nucleus accumbens in mediating reward. J Neurosci 17:8580-8587.

Izzo E, Orsini C, Koob GF, Pulvirenti L (2001) A dopamine partial agonist and antagonist block amphetamine self-administration in a progressive ratio schedule. Pharmacol Biochem Behav 68:701-708.

Joyce EM, Stinus L, Iversen SD (1983) Effect of injections of 6-OHDA into either nucleus accumbens septi or frontal cortex on spontaneous and drug-induced activity. Neuropharmacology 22:1141-1145.

Joyce JN (1991a) Differential response of striatal dopamine and muscarinic cholinergic receptor subtypes to the loss of dopamine. I. Effects of intranigral or intracerebroventricular 6-hydroxydopamine lesions of the mesostriatal dopamine system. Exp Neurol 113:261-276.

Joyce JN (1991b) Differential response of striatal dopamine and muscarinic cholinergic receptor subtypes to the loss of dopamine. II. Effects of 6-hydroxydopamine or colchicine microinjections into the VTA or reserpine treatment. Exp Neurol 113:277-290.

Kelley AE, Smith-Roe SL, Holahan MR (1997) Response-reinforcement learning is dependent on $\mathrm{N}$-methyl-D-aspartate receptor activation in the nucleus accumbens core. Proc Natl Acad Sci USA 94:12174-12179.

Kelly PH, Iversen SD (1976) Selective 6-OHDA-induced destruction of mesolimbic dopamine neurons: abolition of psychostimulant-induced locomotor activity in rats. Eur J Pharmacol 40:45-56.

Kelly PH, Seviour PW, Iversen SD (1975) Amphetamine and apomorphine responses in the rat following 6-OHDA lesions of the nucleus accumbens septi and corpus striatum. Brain Res 94:507-522.

Koob GF, Sanna PP, Bloom FE (1998) Neuroscience of addiction. Neuron 21:467-476.

Laviolette SR, Nader K, van der Kooy D (2002) Motivational state determines the functional role of the mesolimbic dopamine system in the mediation of opiate reward processes. Behav Brain Res 129:17-29.

Lyness WH, Friedle NM, Moore KE (1979) Destruction of dopaminergic nerve terminals in nucleus accumbens: effect on D-amphetamine selfadministration. Pharmacol Biochem Behav 11:553-556.

Mackey WB, van der Kooy D (1985) Neuroleptics block the positive reinforcing effects of amphetamine but not of morphine as measured by place conditioning. Pharmacol Biochem Behav 22:101-105.

Maldonado-Irizarry CS, Kelley AE (1995) Excitotoxic lesions of the core and shell subregions of the nucleus accumbens differentially disrupt body weight regulation and motor activity in rat. Brain Res Bull 38:551-559.

Nader K, van der Kooy D (1997) Deprivation state switches the neurobiological substrates mediating opiate reward in the ventral tegmental area. J Neurosci 17:383-390.

Ogren SO, Archer T, Johansson C (1983) Evidence for a selective brain noradrenergic involvement in the locomotor stimulant effects of amphetamine in the rat. Neurosci Lett 43:327-331.

Parkinson JA, Olmstead MC, Burns LH, Robbins TW, Everitt BJ (1999)
Dissociation in effects of lesions of the nucleus accumbens core and shell on appetitive Pavlovian approach behavior and the potentiation of conditioned reinforcement and locomotor activity by D-amphetamine. J Neurosci 19:2401-2411.

Paxinos G, Watson C (1997) The rat brain in stereotaxic coordinates, Ed 3. San Diego: Academic.

Phillips GD, Robbins TW, Everitt BJ (1994) Bilateral intra-accumbens selfadministration of D-amphetamine: antagonism with intra-accumbens SCH-23390 and sulpiride. Psychopharmacology 114:477-485.

Pierce RC, Kalivas PW (1995) Amphetamine produces sensitized increases in locomotion and extracellular dopamine preferentially in the nucleus accumbens shell of rats administered repeated cocaine. J Pharmacol Exp Ther 275:1019-1029.

Pijnenburg AJ, Honig WM, Van Rossum JM (1975) Inhibition of D-amphetamine-induced locomotor activity by injection of haloperidol into the nucleus accumbens of the rat. Psychopharmacologia 41:87-95.

Pijnenburg AJ, Honig WM, van der Heyden JA, Van Rossum JM (1976) Effects of chemical stimulation of the mesolimbic dopamine system upon locomotor activity. Eur J Pharmacol 35:45-58.

Pradhan AA, Cumming P, Clarke PBS (2002) [ ${ }^{125}$ I] epibatidine-labeled nicotinic receptors in the extended striatum and cerebral cortex: lack of association with serotonergic afferents. Brain Res 954:227-236.

Redgrave P, Prescott TJ, Gurney K (1999) Is the short-latency dopamine response too short to signal reward error? Trends Neurosci 22:146-151.

Robbins TW, Roberts DC, Koob GF (1983) Effects of D-amphetamine and apomorphine upon operant behavior and schedule-induced licking in rats with 6-hydroxydopamine-induced lesions of the nucleus accumbens. J Pharmacol Exp Ther 224:662-673.

Roberts DCS, Zis AP, Fibiger HC (1975) Ascending catecholamine pathways and amphetamine-induced locomotor activity: importance of dopamine and apparent non-involvement of norepinephrine. Brain Res 93:441-454.

Roberts DCS, Corcoran ME, Fibiger HC (1977) On the role of ascending catecholaminergic systems in intravenous self-administration of cocaine. Pharmacol Biochem Behav 6:615-620.

Robledo P, Maldonado R, Koob GF (1993) Neurotensin injected into the nucleus accumbens blocks the psychostimulant effects of cocaine but does not attenuate cocaine self-administration in the rat. Brain Res 622:105-112.

Schultz W (2002) Getting formal with dopamine and reward. Neuron 36:241-263.

Shippenberg TS, Bals-Kubik R, Herz A (1993) Examination of the neurochemical substrates mediating the motivational effects of opioids: role of the mesolimbic dopamine system and D-1 vs. D-2 dopamine receptors. J Pharmacol Exp Ther 265:53-59.

Spyraki C, Fibiger HC, Phillips AG (1982) Dopaminergic substrates of amphetamine-induced place preference conditioning. Brain Res 253:185-193.

Spyraki C, Fibiger HC, Phillips AG (1983) Attenuation of heroin reward in rats by disruption of the mesolimbic dopamine system. Psychopharmacology 79:278-283.

Ventura R, Cabib S, Alcaro A, Orsini C, Puglisi-Allegra S (2003) Norepinephrine in the prefrontal cortex is critical for amphetamine-induced reward and mesoaccumbens dopamine release. J Neurosci 23:1879-1885.

Vezina P, Stewart J (1987) Conditioned locomotion and place preference elicited by tactile cues paired exclusively with morphine in an open field. Psychopharmacology 91:375-380.

Weiner I, Gal G, Rawlins JN, Feldon J (1996) Differential involvement of the shell and core subterritories of the nucleus accumbens in latent inhibition and amphetamine-induced activity. Behav Brain Res 81:123-133.

West CH, Boss-Williams KA, Weiss JM (1999) Motor activation by amphetamine infusion into nucleus accumbens core and shell subregions of rats differentially sensitive to dopaminergic drugs. Behav Brain Res 98:155-165.

Wise RA (2002) Brain reward circuitry: insights from unsensed incentives. Neuron 36:229-240.

Yokel RA, Wise RA (1975) Increased lever pressing for amphetamine after pimozide in rats: implications for a dopamine theory of reward. Science 187:547-549.

Yokel RA, Wise RA (1976) Attenuation of intravenous amphetamine reinforcement by central dopamine blockade in rats. Psychopharmacology 48:311-318.

Zahm DS, Brog JS (1992) On the significance of subterritories in the "accumbens" part of the rat ventral striatum. Neuroscience 50:751-767. 